\title{
Game traffic analysis: An MMORPG perspective is
}

\author{
Kuan-Ta Chen ${ }^{\text {a,c }}$, Polly Huang ${ }^{\text {a,b }}$, Chin-Laung Lei ${ }^{\text {a,b,* }}$ \\ a Department of Electrical Engineering, National Taiwan University, Taiwan, ROC \\ ${ }^{\mathrm{b}}$ Graduate Institute of Networking and Multimedia, National Taiwan University, Taiwan, ROC \\ ${ }^{\mathrm{c}}$ Institute of Information Science, Academia Sinica, Taiwan, ROC
}

Received 5 September 2005; received in revised form 16 November 2005; accepted 25 November 2005

Available online 27 December 2005

Responsible Editor: Nelson Fonseca

\begin{abstract}
Online gaming is one of the most profitable businesses on the Internet. Of all the genres of online games, MMORPGs (Massive Multiplayer Online Role Playing Games) have become the most popular among network gamers, and now attract millions of users who play in an evolving virtual world simultaneously over the Internet. To gain a better understanding of game traffic and contribute to the economic well-being of the Internet, we analyze a 1356-million-packet trace from a sizeable MMORPG called ShenZhou Online. This work is, as far as we know, the first formal analysis of MMORPG server traces.

We find that MMORPG and FPS (First-Person Shooting) games are similar in that they both generate small packets and require low bandwidths. In practice, the bandwidth requirement of MMORPGs is the lower of the two due to less realtime game playing. More distinctive features are the strong periodicity, temporal locality, irregularity, and self-similarity observed in MMORPG traffic. The periodicity is due to a common practice in game implementation, where game state updates are accumulated within a fixed time window before transmission. The temporal locality in game traffic is largely due to the game's nature, whereby one action leads to another. The irregularity, which is unique to MMORPG traffic, is due to the diversity of the game's design so that the behavior of users can vary drastically, depending on the quest at hand. The self-similarity of the aggregate traffic is due to the heavy-tailed active/idle activities of individual players. Moreover, we show that the arrival of game sessions within $1 \mathrm{~h}$ can be modelled by a Poisson model, while the duration of game sessions is heavy-tailed.
\end{abstract}

(C) 2005 Elsevier B.V. All rights reserved.

Keywords: Internet measurement; MMORPG; Network games; Traffic analysis

\footnotetext{
A preliminary version of this paper [1] appeared in the Proceedings of the International Workshop on Network and Operating Systems Support for Digital Audio and Video (NOSSDAV 2005). This research is supported in part by the National Science Council of the Republic of China under grant NSC 94-2213-E-002-043.

* Corresponding author. Address: Department of Electrical Engineering, National Taiwan University, BL-604, No. 1, Section 4, Roosevelt Road, Taipei 106, Taiwan. Tel.: +8862 33663582; fax: +886223638247.

E-mail addresses: jethro@fractal.ee.ntu.edu.tw (K.-T. Chen), phuang@cc.ee.ntu.edu.tw (P. Huang), lei@cc.ee.ntu.edu.tw (C.-L. Lei).
} 


\section{Introduction}

MMORPGs (Massive Multiplayer Online Role Playing Games) have become extremely popular among network gamers, millions of whom now play in an evolving virtual world simultaneously over the Internet. The number of active player subscriptions doubled between July 2004 and June 2005 to a 500million player base [2]. Thus, the amount of game traffic on the Internet has become increasingly significant. According to a report on backbone traffic analysis [3], about $3-4 \%$ of the traffic can be attributed to six popular games. A better understanding of game traffic is vital, given its significant share of overall traffic and the dissimilar nature of games to dominant Internet applications such as the World Wide Web, peer-to-peer file sharing, and streaming.

We focus on MMORPGs for two reasons. First, they are now the most prominent genre of online games, especially in Asia. In Taiwan, Gamania ${ }^{1}$ (the operator of the popular game Lineage ${ }^{2}$ ) owns more than $4000 \mathrm{Mbps}$ of dedicated links ${ }^{3}$ for game traffic. According to Game Flier, ${ }^{4}$ the top game, Ragnarok Online, claimed a record of 370,000 users playing online simultaneously in December, 2004; about $1.5 \%$ of Taiwan's population. The second reason is that most MMORPGs exchange messages using TCP. Although TCP is not designed for real-time communication, it is not yet clear whether it is unsuitable for the MMORPG traffic transmission. To the best of our knowledge, this work is the first to analyze and characterize MMORPG traffic.

We trace ShenZhou Online [4], a commercial, mid-sized MMORPG, and analyze a 1,356-million-packet trace. We thoroughly analyze the game's traffic from a number of aspects: traffic patterns within individual connections, the characteristics of aggregate traffic, and session characteristics. Our analysis focuses primarily on the temporal dependence of traffic processes, periodicity, and variability, and the physical explanations and implications of such effects. The major findings can be summarized as follows:

\footnotetext{
${ }^{1}$ Gamania, http://www.gamania.com/.

2 NCsoft Corporation, http://www.lineage.com/.

3 TWNIC, http://map.twnic.net.tw/.

${ }^{4}$ Game Flier, http://www.gameflier.com/.
}

- The average bandwidth requirement per client is about $7 \mathrm{Kbps}$, which is much lower than the $40 \mathrm{Kbps}$ average observed for Counter-Strike [5]. We believe the lower bandwidth requirement is due to the relatively slow pace of MMORPGs.

- Traffic in both directions exhibits short-term temporal dependence within connections. In client traffic (traffic from the client to the server), the dependence is due to the clustering nature of user actions. The same effect in server traffic is caused by the spatial locality of the number of characters near the player character, which shows up in terms of the temporal locality in traffic as the player character moves continuously on the map.

- Temporal dependence still exists in aggregate client traffic. We believe this is due to the global events in games, which in turn cause the "flash crowd" effect. Furthermore, the aggregate traffic in both directions exhibits strong periodicity, which implies that game processing for all clients is synchronized. Packet bursts due to the periodic state update mechanism do cause network performance problems, i.e., superfluous packet loss.

- Game traffic burstiness is much less than in TELNET traffic, for both individual connections and aggregate traffic. At the same time, the aggregate game traffic exhibits self-similarity over a wide range of time scales (for at least $2 \mathrm{~h}$ ), which could be explained by the heavy-tailed activelidle periods of individual players.

- The arrival of game sessions within $1 \mathrm{~h}$ can be modelled by a homogeneous Poisson model. The duration of both game and map sessions is shown to be heavy-tailed, which could be a consequence of the game's nature.

For MMOGs, although the bandwidth requirement of each game client might be small, the number of concurrent connections could be very large. For instance, the record of 370,000 players online simultaneously (Ragnarok Online, Taiwan, 2004) implies that at least $3.7 \mathrm{Gbps}$ of bandwidth is required, assuming each client needs $10 \mathrm{Kbps}$ on average. Furthermore, according to our analysis, the traffic of concurrent connections to a game are positively correlated, rather than independent, and the aggregate game traffic exhibits the self-similar property; therefore, the instantaneous peak rate can be much higher than the estimated average rate. For the above reasons, we believe that understanding game traffic is especially critical for network planning at the game server side and not negligible 
for the game providers and ISPs as the online game population continues to increase rapidly [2].

In remainder of this paper, we discuss these issues in detail. Section 2 describes related works and the game ShenZhou Online. We discuss the trace measurement methodology and trace summary in Section 3. In Section 4, we analyze the traffic patterns of individual connections. We then investigate the behavior of aggregate traffic in Section 5. Next, in Section 6, we analyze the session-level characteristics of both TCP connections and game sessions. Finally, in Section 7, we present our conclusions.

\section{Background}

\subsection{Related work}

As network games are becoming major contributors to overall Internet traffic, a great deal of effort is being devoted to the analysis and modeling of network game traffic. Bangun et al. [6] analyzed a network trace captured at an Internet café for two LAN games, Quake and Starcraft. Their work focused on how payload sizes and inter-packet times vary with the number of players. Later, Bangun and Dutkiewicz proposed models of payloads and interpacket times based on a trace of another LAN game called Starsiege Tribes. Borella proposed source models for the popular FPS (First-Person Shooting) game Quake, where the packet interarrival times and packet sizes are modelled as extreme distribution, exponential distribution, or deterministic [7]. A similar work by Färber characterized traffic for another FPS game called Counter-Strike [8].

Feng et al. [5] analyzed a 500-million-packet trace of a busy Counter-Strike server. Their study revealed that game traffic is highly predictable; however, it contains bursts of tiny packets. Though MMORPGs and FPS games are very different game genres, we find they are similar in terms of packet size and periodicity. In [5], the authors further evaluated the impact of tiny packets on network infrastructures by a pressure test on a commercial off-the-shelf NAT device. The test indicated that the device cannot handle such packets well.

\subsection{About ShenZhou Online}

ShenZhou Online is a mid-scale, commercial MMORPG that is popular in Taiwan [4], where there are thousands of players online at any one time. To play, users purchase "game points" from

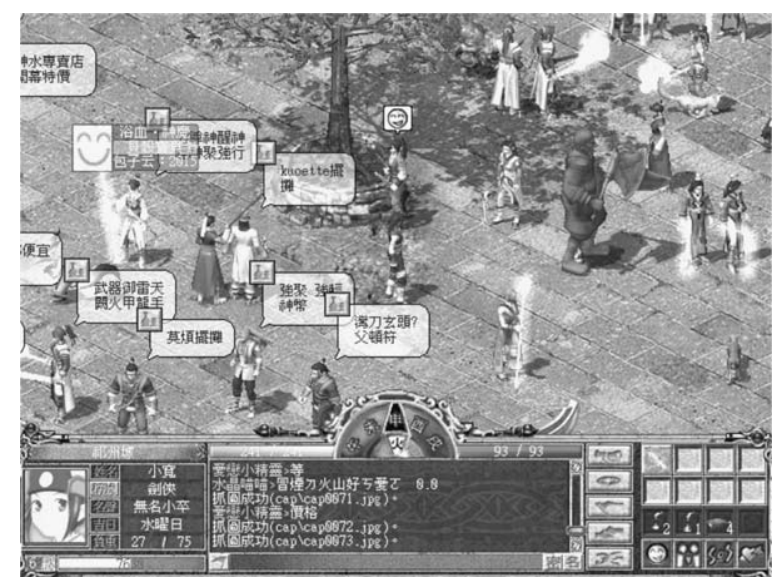

Fig. 1. A screen shot of ShenZhou Online.

a convenience store or online. A screen shot of ShenZhou Online is shown in Fig. 1. The character played by the author is the man in the center of the screen with a smiling face above him. He is in a typical marketplace, where the other players keep stalls. As is normal in MMORPGs, a player can engage in fights with the other players or with random creatures, train himself for special skills, participate in marketplace commerce, or take on a quest.

\section{Trace collection}

With the help of the ShenZhou Online staff, we set up a traffic monitor beside the game servers. The monitor was attached to a layer-4 switch upstream of the LAN containing the game servers (we call it the "game LAN"). The port forwarding capability of the tapped layer-4 switch was enabled so that all inbound/outbound game traffic was forwarded to our monitor as a copy. To minimize the impact of monitoring, all remote management operations were conducted via an additional network path, i.e., the game traffic and management traffic did not interfere with each other. The network topology and setup of the game servers and the traffic monitor are shown in Fig. 2.

The traffic monitor was a FreeBSD PC equipped with $1.5 \mathrm{GHz}$ Pentium 4 and $256 \mathrm{MB}$ RAM. We used tcpdump [9] with the kernel built-in BPF to obtain traffic traces. Because of the restrictions of the network topology, the switch forwarded all traffic sent to and from the game LAN, including nongame-playing traffic like HTTP, DNS, and SMB packets. These unwanted traffic types were filtered out using the filtering support of tcpdump. Out of 


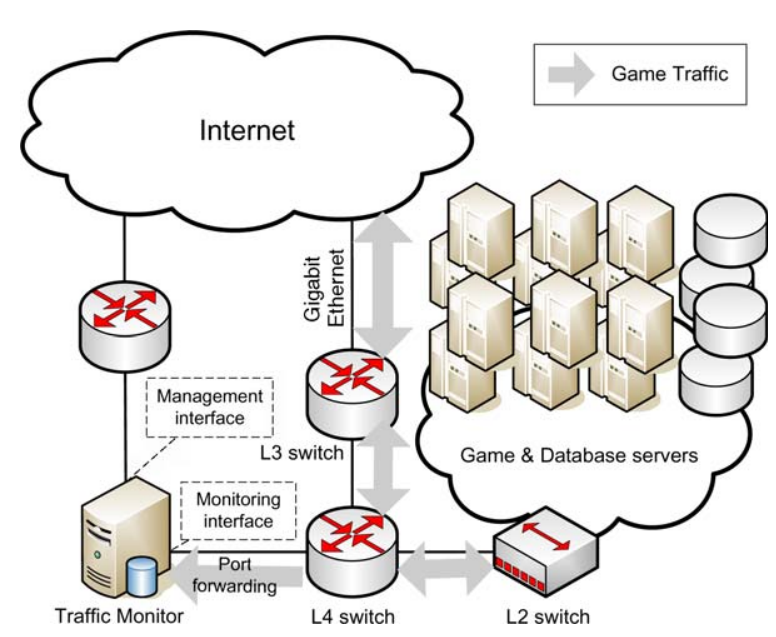

Fig. 2. Network setup for traffic measurement.

consideration for data privacy and storage, only IP and TCP headers were recorded.

We randomly chose a subset of game sets in each trace, and only packets belonging to the selected game sets were logged. A game set, which is logically a "game server" from a player's viewpoint, comprises an entry server, several map servers, and a database server. All game sets are equivalent in content, but isolated. The reason for providing identical game sets is to distribute the workload over a number of servers with limited game content, e.g., terrain, missions, and creatures in the virtual world. We took two packet traces, $\mathscr{N}_{1}$ and $\mathscr{N}_{2}$, which recorded traffic for two and three game sets, respectively. The traces, summarized in Table 1 , spanned 8 and $12 \mathrm{~h}$, respectively, and contained more than 1356 million packets in total. In the packet-level analysis presented in this paper, except for packets (less than 10) discarded due to TCP checksum errors, all recorded game packets are used. The user authentication process is through separate TCP connections and ports; thus, nonplaying activities, such as login attempts and subscription status validation, are not included in our analysis. On the other hand, for session-level analy- sis (Section 6), we treat a TCP connection as a legitimate map session if it satisfies the following conditions: (1) its presence time (observed by our monitor) is longer than $10 \mathrm{~s}$; and (2) it comprises at least 10 packets in both directions. No connections were trimmed, even if some of them were partially observed by the monitor.

\section{Traffic characteristics of individual connections}

In this section, we investigate traffic patterns embedded in individual connections. We first examine the size of packets sent by either game clients or servers, and then analyze the packet load and bandwidth usage of each client. Next, we search for patterns in the inter-packet times within each connection. Lastly, the peak rate and traffic burstiness over a range of time scales are investigated.

For the sake of brevity, we denote packets sent by game clients, including data packets and TCP acknowledgement packets, as "client packets", and all traffic sent by clients as "client traffic." The terms "server packets" and "server traffic" are similarly defined.

\subsection{Packet size}

Fig. 3 shows the cumulative distribution function (CDF) of the payload size, which is the packet size without the TCP/IP header of 40 bytes. Pure TCP ack packets do not count. As the figure shows, client and server packets are drastically different in payload size. The discrepancy conforms to our intuition, since client packets contain one player's commands, while server packets convey nearby characters' actions and states as well as system messages. The client packets are extremely small: $98 \%$ of client packets have a payload size smaller than 32 bytes. The two modes 23 and 27 bytes, which comprise $36 \%$ and $52 \%$ of packets respectively, show that user actions are dominated by a few popular commands, such as "walk" and "attack." On the other hand, server packets have a much wider

Table 1

Summary of game traffic traces

\begin{tabular}{llllllll}
\hline ID & Date & Time & Period (h) & Drops $^{\mathrm{a}}$ & Connections (Cens.) & Pkt. (in/out) & Bytes (in/out) \\
\hline $\mathscr{N}_{1}$ & $8 / 29 / 04$ & $15: 00$ & 8 & $0.003 \%$ & $57,945(6.5 \%)$ & $342 \mathrm{M} / 353 \mathrm{M}$ & $4.7 \mathrm{~TB} / 27.3 \mathrm{~TB}$ \\
$\mathscr{N}_{2}$ & $8 / 30 / 04$ & $13: 00$ & 12 & $?^{\mathrm{b}}$ & $54,424(3.5 \%)$ & $325 \mathrm{M} / 336 \mathrm{M}$ & $4.7 \mathrm{~TB} / 21.7 \mathrm{~TB}$ \\
\hline
\end{tabular}

a This column gives the kernel drop count reported by tcpdump.

b The reported kernel drop count was zero, but we actually found some packets are dropped. 


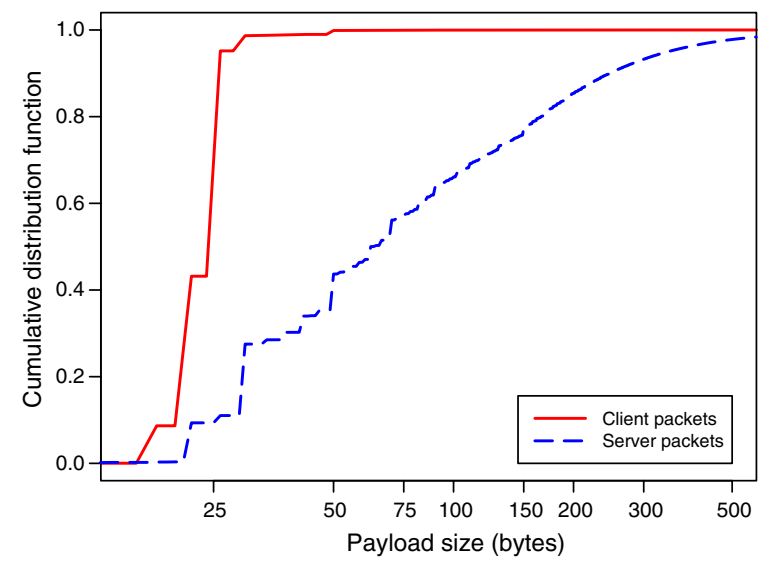

Fig. 3. Payload size distribution.

distribution with an average payload size of 114 bytes. The payload size distribution generally agrees with the finding in [5] that the packet size in game traffic is generally much smaller than has been observed at Internet exchange points, where the mean packet size is above 400 bytes [3].

According to the delay ack option [10], ack packets are sent for alternate TCP segments received on a connection, unless the delayed ack timer (usually set to $200 \mathrm{~ms}$ ) has expired. Thus, in ideal circumstances, the ratio of pure ack packets should take up approximately one-third. However, $38 \%$ of the packets in our trace are pure TCP acks. We find that this relatively high ratio is due to the fact that game packets are generated at a low rate, so it is probable that no further packets will arrive before the expiration of the delayed ack timer. Furthermore, for overall client traffic, we can calculate the overhead of TCP/IP headers and TCP acks by the ratio of bytes used. The cost is noteworthy: TCP/IP headers comprise $73 \%$, where pure TCP acknowledgement packets are responsible for $30 \%$. Note that the former includes the latter. The average packet size is 84 bytes if we count the overall game traffic. While routers and other network devices are often designed on the assumption that the average packet size is between 125 and 250 bytes [11], the popularity of online games may challenge the processing power of network devices because of the large number of tiny game packets.

\subsection{Packet load and bandwidth usage}

To determine the bandwidth usage of each game client, we compute the average packet load and average throughput rate for each connection. As shown in Figs. 4 and 5, the packet load and bandwidth needed to play an MMORPG are surprisingly low. For most connections, the average server packet rates are lower than $5 \mathrm{pkt} / \mathrm{s}$. Even by considering TCP acks, $99 \%$ of connections have a packet load less than $15 \mathrm{pkt} / \mathrm{s}$ in both directions.

With the low packet rate and small payload, the bandwidth requirement of an MMORPG is even lower than the narrowest last-mile link, $56 \mathrm{Kbps}$ modem. Nearly all connections consume less than $3 \mathrm{Kbps}$ for client data packets, and less than $8 \mathrm{Kbps}$ when TCP acks are considered. Although server traffic uses more bandwidth, only $7 \mathrm{Kbps}$ is required on average. The overall bandwidth usage is much lower than the average $40 \mathrm{Kbps}$ needed by Counter-Strike [5]. We believe the difference is due to the game's nature; that is, MMORPGs are relatively slow-paced compared to FPS games, which usually

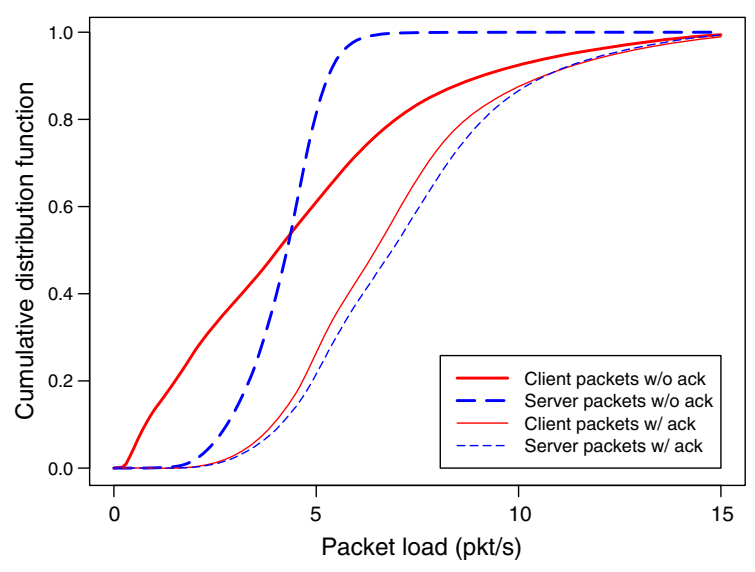

Fig. 4. Packet load distribution.

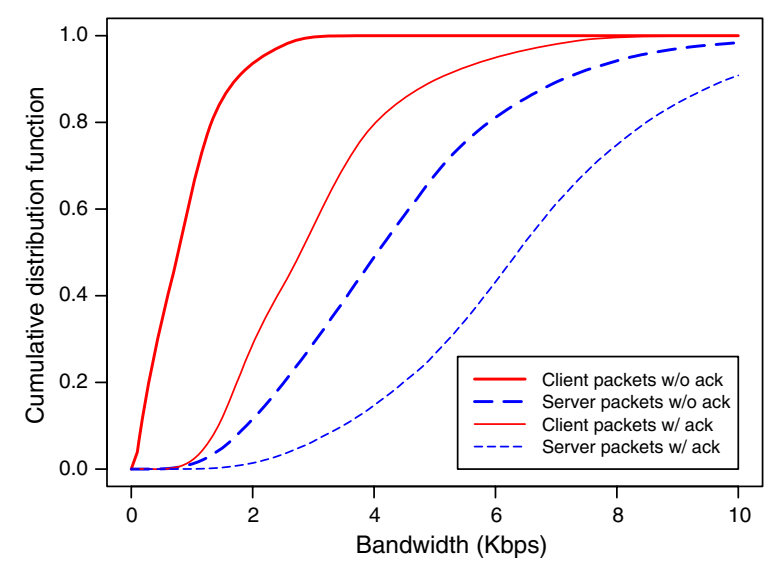

Fig. 5. Bandwidth distribution. 
require players to make sub-second decisions. On the other hand, the bandwidth usage of MMORPG is comparable to that of the online RTS (Realtime Strategy) game called Warcraft III [12]. Indeed, the pace of gaming in MMORPGs and RTS games is similar, while the pace of FPS games is much faster.

\subsection{Distribution of packet interarrival times}

In order to understand traffic dynamics within individual connections, we first observe the distribution of inter-packet times for client data packets. As Fig. 6 shows, most packet interarrival times are spread over $0-600 \mathrm{~ms}$. We find that the best-fit exponential distribution with a rate of $8 \mathrm{pkt} / \mathrm{s}$ approximately fits the empirical cumulative distribution function; however, the deviation of the exponential-fit is apparent at time scales larger than $200 \mathrm{~ms}$. Further investigation shows that the deviation from the exponential distribution of inter-packet times is caused by the diversity of user behavior, which is a distinct feature of adventureoriented games, especially MMORPGs.

For game genres like FPS, RTS, and FTG (Fighting Games), during a game, players must be active continuously, or they will be defeated. Such games are usually round-based so that players are forced to be idle if they are defeated, but they can be active again in the next round. In contrast, MMORPGs and other adventure-oriented games do not require participants to "play" all the time. During a game, players can do anything at will: they can carefully inspect their equipment; wait somewhere for friends or some event; or simply leave

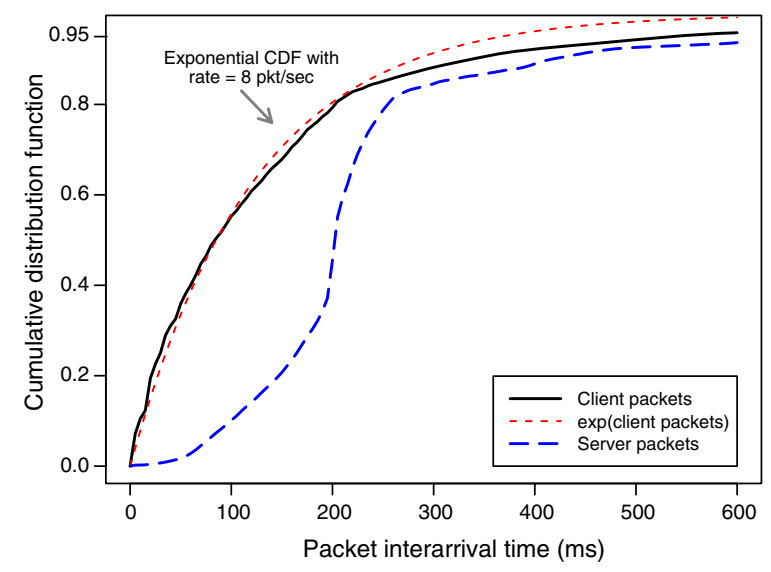

Fig. 6. Packet interarrival distribution. their characters idle, unless the characters are on the battlefield. We found that most players were idle, at least for a while, during the trace period, and some were even idle most of the time. To demonstrate, we picked three representative connections from the traces, namely, active sessions (almost no idle time), idle sessions (almost always idle), and regular sessions. The distributions of packet interarrival times of these sessions are shown in Fig. 7. We observe that the distribution of the active session is nearly exponential, while that of the idle session is far from exponential. Actually, the latter is a hybrid of a uniform distribution and a deterministic distribution, where the determination derives from the 5$\mathrm{s}$ interval of the keep-alive timers. Naturally, the inter-packet time distribution of the regular session is between the above two extremes. This explains the distribution in Fig. 6, which is close to exponential in small time scales, but has a longer tail, since it comprises inter-packet times from sessions with various degrees of activity. Furthermore, the diversity of user behavior makes it difficult, if not impossible, to define a typical player so that modeling user behavior and source traffic in MMORPGs is especially challenging.

On the other hand, server packet interarrivals are much more regular-approximately $50 \%$ of the interarrival times are around $200 \mathrm{~ms}$. The concentration of inter-packet times at certain intervals shows that server processing is round-based, i.e., servers broadcast information to clients on a regular basis. We investigate the periodicity of game traffic further in Section 5.2.

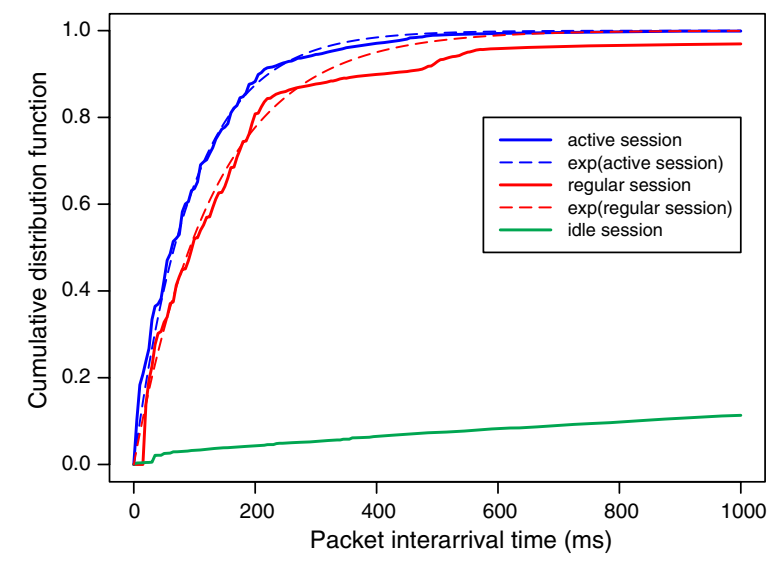

Fig. 7. Client packet interarrival distributions for representative sessions. 


\subsection{Temporal dependence of packet interarrivals}

As the interarrival times of data packets sent by game clients are close to exponential, at least for active sessions, one may wonder if player commands conform to Poisson arrivals. An initial check via the auto-correlation functions (ACF), shown in Fig. 8, indicates that packet interarrivals in both directions exhibit positive temporal dependence. Thus, the hypothesis of Poisson input is rejected.

In Fig. 8(a), client packet interarrival times show positive auto-correlations up to $1 \mathrm{~min}$. We believe this phenomenon is attributable to the clustering nature of player actions; that is, commands issued by players are often successive and made in bursts. For example, common behavior of a character in a game includes walking, chatting, resting, fighting, examining loot, trading, and so forth. Whatever the type of action, it is likely to last for a period of tens of seconds. Furthermore, traffic sent by a game client is decided by the type of current action. For example, when a player is talking with other players, examining equipment, or conducting a business transaction, the client sends very little traffic. In contrast, fighting and movement actions result in bursts of commands, which generate bursts of client packets. To demonstrate, 2000 successive interpacket times drawn from a randomly selected session are depicted in Fig. 9. We observe that the inter-packet times of similar intervals are clustered, where clusters of approximately $333 \mathrm{~ms}(1 / 3 \mathrm{~s})$ are

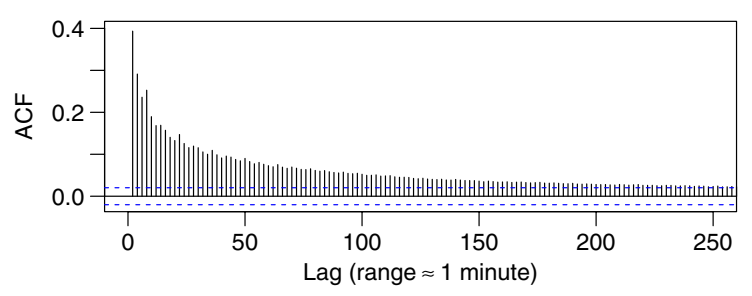

(a) ACF for client packets

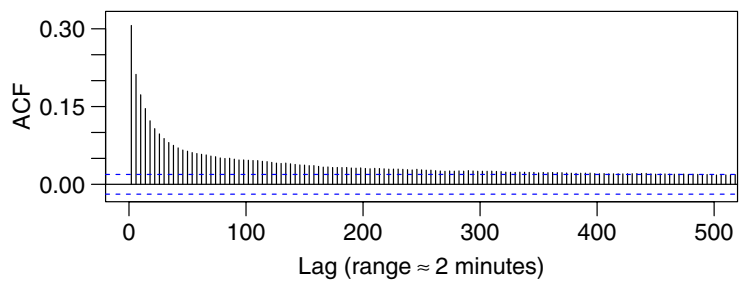

(b) ACF for server packets

Fig. 8. Correlograms of within-connection packet interarrivals.

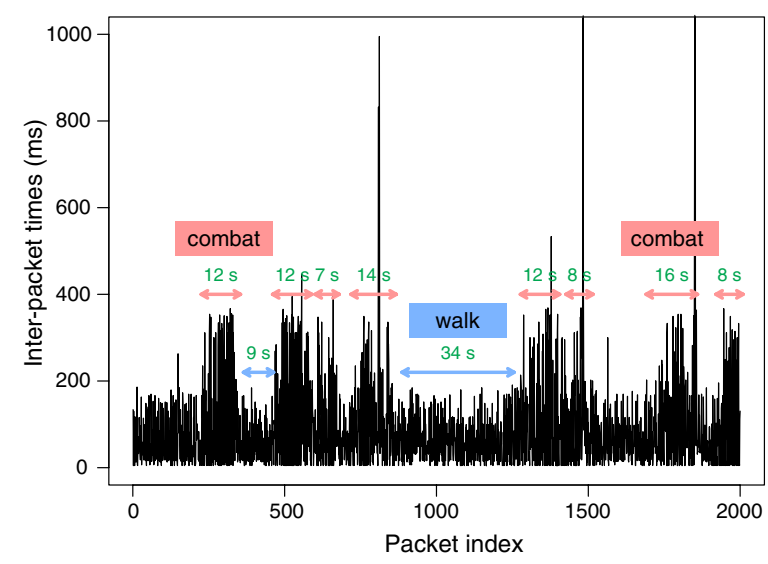

Fig. 9. The clustering nature of player actions.

identified as fighting actions, i.e., each command corresponds to a strike action. Similarly, clusters of approximately $167 \mathrm{~ms}(1 / 6 \mathrm{~s})$ are identified as movement actions, i.e., each command moves the character one step further. Since player actions exhibit the above-mentioned temporal locality and the rate of client packets depends on the type of current action, positive auto-correlations (up to $1 \mathrm{~min}$ ) are formed in client traffic, as shown in Fig. 8(a).

In contrast, server packet interarrivals exhibit positive auto-correlations up to $2 \mathrm{~min}$. We explain the phenomenon by the following observations. First, server packets primarily convey the position and state updates of characters, including the player character (the character controlled by the player himself), nearby characters played by other gamers, and non-player characters (NPC) controlled by the game logic. We use "neighbors" to denote characters around the player character, no matter if they are NPCs or belong to other gamers. Based on our observations in Section 4.3, we find that servers notify each client about the most up-to-date positions of his neighbors on a per-round basis. More specifically, according to the developers of ShenZhou Online, servers send out position updates in each round, unless no other characters are around, or no neighbors have moved since the last position update was sent. In other words, the position of each neighbor is only included in the server update message if it has changed since the last update. This design suggests that the rate of position updates would be roughly proportional to the number of neighbors if the movements of each character's neighbors do not follow any particular pattern. We confirm this conjecture by a separate experiment conducted 
at the client side. In the experiment, we compute the average rate of server data packets received by the game client for different scenarios in which varying densities of neighbors are around the player character. Each scenario lasted for $30 \mathrm{~min}$. The average server data packet rates we observed were $2.8,6.2$, and $10.8 \mathrm{pkt} / \mathrm{s}$ when the character was in a quiet place, a unpopular intersection, and a crowded market, respectively. The above conjecture is therefore confirmed by the approximate proportionality between the server packet rate and neighbor density.

Second, because the game map is continuous and characters are widely spread over the map, the number of neighbors across the map has the property of spatial locality. That is, two characters that are geographically close to each other have a similar number of neighbors. Furthermore, when a character moves across the map continuously, the spatial locality of the number of neighbors will be transformed to the temporal locality of the number of neighbors, i.e., the time series formed by the number of neighbors sampled in each round is auto-correlated. An exception to spatial continuity (and therefore temporal locality) is that a character can "teleport" to another place instantly by a scroll or magic; however, compared with walking actions, this feature is not used frequently.

In summary, we observe that (1) the number of neighbors possesses temporal locality; (2) the rate of position updates is roughly proportional to the number of neighbors; and (3) server packets primarily convey position updates. As a consequence of the above interrelation between server mechanisms and user behavior, temporal dependence up to $2 \mathrm{~min}$ is formed in server packet interarrivals, as shown by the auto-correlation function in Fig. 8(b).

\subsection{Peak rate and burstiness}

In Section 4.2, we noted that the "average" packet load of each connection is not high; however, in terms of network performance, the "instantaneous" rate is more relevant. Although we cannot actually obtain an instantaneous rate of a measured traffic process, we can approximate it by computing the peak rate at a certain (usually small) time scale. Unlike the average rate, the peak rate can provide information about how much bandwidth a connection would need in the most demanding cases.

In Fig. 10(a), we plot the distribution of the peak packet load measured at two time scales, $0.1 \mathrm{~s}$ and $10 \mathrm{~s}$, for client traffic and server traffic, respectively. We can see that the peak rate of client traffic is generally higher than that of server traffic. For most connections, the peak rate is within 5-7 packets for client traffic and within 2-3 packets for server traffic within $0.1 \mathrm{~s}$. Compared with the average rate of less than 0.5 packets per $0.1 \mathrm{~s}$ for traffic in both directions, this measure shows that the release of packets is not smooth over sub-second time scales. At the same time, game clients may send out 200 packets within $10 \mathrm{~s}$, compared with the average of 45 packets for the same period. This measure shows that the variability of traffic remains in intervals of $10 \mathrm{~s}$. On the other hand, the maximum number of packets sent by game servers within $10 \mathrm{~s}$ is exactly 50 for most connections, which again supports the observation on the regularity of server traffic in Section 4.3.

In Section 4.4, we observed that both client traffic and server traffic tend to be temporally clustered. This temporal dependence in inter-packet times definitely makes traffic less smooth, i.e., more bursty. To understand traffic burstiness, i.e., the variability

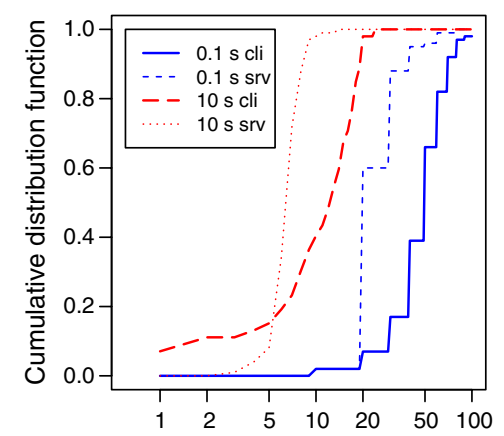

(a) Packet load (pkt/s)

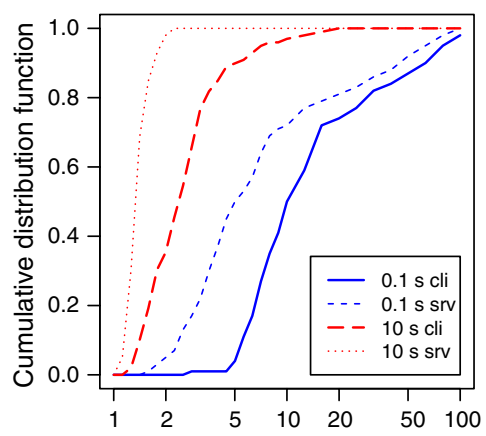

(b) Peak to mean ratio

Fig. 10. (a) Peak packet load (pkt/s) and (b) peak to mean ratio of packet load observed in different time scales. 
of packet counts observed in successive periods, of game traffic, we compute the peak-to-mean ratio, which is defined as the ratio of the peak packet rate versus the average packet rate at a certain time scale for each connection. The distributions of the peakto-mean ratio for individual connections are shown in Fig. 10(b). We find that the peak-to-mean ratio of traffic in either direction can be at most 100:1 at $0.1 \mathrm{~s}$; however, on average, it is $14: 1$ and $5: 1$ for client traffic and server traffic, respectively. At $10 \mathrm{~s}$, the peak-to-mean ratio is reduced to an average of 2.5:1 for client traffic, and 1.4:1 for server traffic. Nevertheless, we cannot determine whether game traffic is "bursty" simply by the magnitude of the peakto-mean ratio, as "how bursty is bursty?" is not a trivial problem. In the following, we seek to answer the question by comparing game traffic with traffic in two other applications: TELNET and FTPDATA.

Unlike the peak-to-mean ratio, the index of dispersion for counts (IDC), which is also an indicator of traffic burstiness, is defined over various time scales. The IDC at time scale $t$ is defined as the variance in the number of arrivals in an interval of time $t$ divided by the mean number of arrivals in $t$ [13], that is,

$I_{t}=\frac{\operatorname{Var}\left(N_{t}\right)}{E\left(N_{t}\right)}$,

where $N_{t}$ indicates the number of arrivals in an interval of time $t$. The IDC is thus defined so that, for a Poisson process, the value of the IDC is 1 for all $t$. We compare the game traffic burstiness to that of TELNET and FTPDATA for several reasons. First, both applications tend to have long-term connections, i.e., the duration is in the scale of minutes or hours, which is usually the case with network games. This explains why we do not include HTTP in the comparison, as most HTTP transactions are extremely short. Second, despite the graphical user interface, client/server network games are very similar to TELNET applications in terms of interactivity and the command-response pattern, where a server responds to client commands by sending back the latest status maintained by the server. Third, though bulk data transfer, i.e., FTPDATA, is very different to the behavior of network games, it can act as a reference because its traffic burstiness arises primarily from the communication protocol, TCP, and the network. That is, unlike network games, where the timing of data packets released into network is reasonably close to the timing of data packets being generated, the timing of FTPDATA packets is dominated by TCP's congestion control mechanisms and the conditions of the network path, such as the round-trip time, hop distance, level of congestion, and cross-traffic.

The TELNET source traffic we use is the $L B L$ TCP3 trace from the Internet Traffic Archive [14], which is a 2-h packet trace containing 322 TELNET sessions. Because no FTP traces with sufficient volume are available in the Internet Traffic Archive, we conducted a 4-h measurement on the gateway router at ee.ntu.edu.tw. The measurement captured only FTPDATA (TCP port 20) packets, and yielded a total of 5.3 million packets and 156 FTPDATA sessions. To avoid being misled by intra-campus file transfers, which usually produce smoother traffic (since congestion is less likely to occur along the path), we removed intra-campus sessions. This left 4.2 million packets and 132 sessions. Hereafter, we call this trace NTU-FTPDATA. Since we are concerned with traffic burstiness of individual TCP connections, we first compute the IDC of each connection, and then compute the average IDC for each application by taking the geometric mean of IDC magnitudes at the same time scales. The reason for using the geometric mean instead of the commonly used arithmetic mean is that the IDCs are actually ratios (variance versus expected value). Taking an arithmetic mean of ratios with different bases could be misleading; hence, the geometric mean is recommended for such cases [15].

Fig. 11 shows the averaged IDC measures of game traffic in both directions, TELNET source traffic, and FTPDATA source traffic. We also provide the IDC of a Poisson process for comparison.

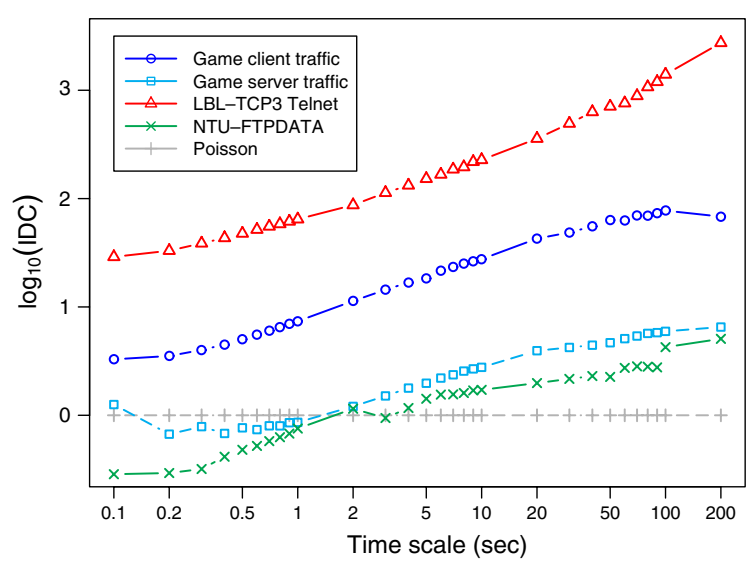

Fig. 11. The traffic burstiness comparison of game traffic, TELNET traffic, and FTPDATA traffic. 
Note that, before computing the IDC, all traffic processes were normalized to the same mean rate to obtain comparable IDC ordinates. Intuitively, the traffic sent by game clients should have similar burstiness to TELNET source traffic; however, in Fig. 11, we find that TELNET traffic is much more bursty than game traffic in both directions. We believe this discrepancy is due to the following reasons. First, human typing speeds can be extremely fast, say, 5-10 keystrokes per second, which is much faster than the number of mouse clicks players can make during the same interval. Second, some built-in automatic steering mechanisms (see Section 5.2) in game clients, such as auto-walk and autoattack, induce a certain regularity into client traffic, thereby reducing the burstiness of the traffic. Third, keep-alive messages, which are sent every $5 \mathrm{~s}$, cut off the possible heavy tail of game packet interarrival times. In contrast, TELNET packet interarrivals have been shown to be heavy-tailed, which increases the burstiness of packet arrival processes [16].

Game server traffic, by comparison, is much less bursty than game client traffic. In particular, the burstiness of server traffic is lower than that of a Poisson process at small time scales $(<2 \mathrm{~s})$, which is the consequence of periodic position updates. Except for the low burstiness at small time scales, the burstiness of server traffic gradually increases in higher time scales as client traffic becomes more bursty. We believe this behavior is due to the fact that part of the server traffic is actually a response to client commands; therefore, its variability is affected by client traffic and becomes more bursty as the latter becomes more bursty at large time scales. Not surprisingly, FTPDATA connections have relatively lower traffic burstiness. The reason is that FTP applications transmit a full window of packets in each round-trip time whenever possible, which leads to strong periodicity at the time scale approximately equal to the roundtrip time. With stable network conditions, the primary factor in traffic fluctuations is TCP's congestion control, i.e., the increase and decrease of the congestion window. Consequently, FTPDATA traffic exhibits relatively lower burstiness, provided that the network conditions of most connections in $N T U$-FTPDATA are stable.

\section{Aggregate traffic characteristics}

We now seek to identify traffic patterns in the aggregate arrival processes of data packets, espe- cially whether within-connection patterns continue to exist and how they change due to flow multiplexing. We first identify the temporal dependence in aggregate traffic and derive a flash-crowd event caused by global events. Next, we identify the periodicity and synchronization rooted in the system's design and show that this degrades network performance. Lastly, we show the self-similarity in game traffic in both directions, and provide physical explanations based on player activities.

To obtain aggregate packet arrivals, we count the number of packets in every $10 \mathrm{~ms}$ for both inbound and outbound traffic. As auto-correlation functions and spectrum are defined on a (second-order) stationary process, we ensure the stationarity of the packet counting time series by sub-sampling. That is, we first select a subset of connections that span a complete 2$\mathrm{h}$ period, and count the packets corresponding to the selected connections during that period.

\subsection{The flash crowd effect}

To understand the patterns in aggregate client traffic, we examine the temporal dependence of

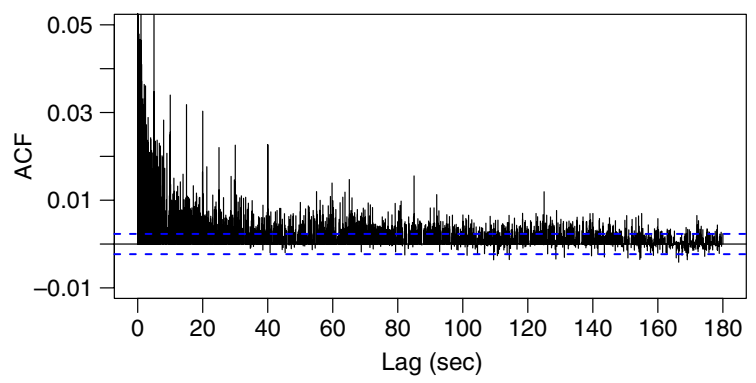

(a) Correlograms of aggregate client packet arrivals

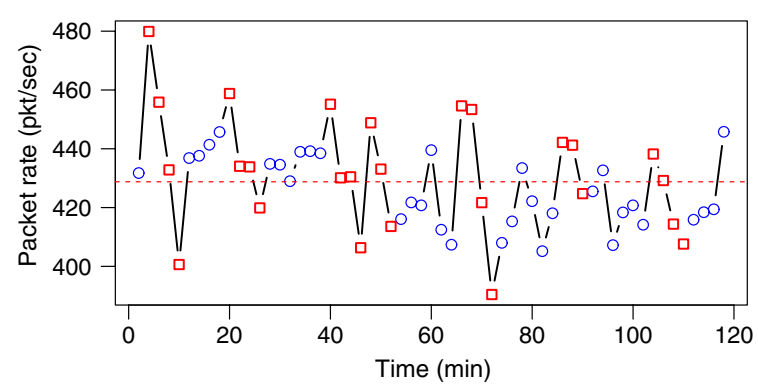

(b) Average packet rates for a certain two-hour period

Fig. 12. The temporal locality in aggregate client traffic and its explanation. Figure (b) plots the average packet rates sampled every $2 \mathrm{~min}$ for a period of $2 \mathrm{~h}$. Peaks, valleys, and their inbetween samples, which are considered to be the effects of global events, are marked by squares. 
aggregate client packet arrivals by correlograms, as shown in Fig. 12(a). From the figure, we can see that client packet arrivals exhibit sustained positive auto-correlations in lags longer than $3 \mathrm{~min}$.

Although we have already seen a similar effect in within-connection packet interarrivals in Section 4.4 , the aggregate traffic exhibits positive auto-correlations of a longer range ( $3 \mathrm{~min}$ versus $1 \mathrm{~min}$ ). While the temporal dependence within individual connections arises from the clustering nature of players' actions, temporal dependence in aggregate packet arrivals initially seems counter-intuitive. In other words, it is unreasonable that a considerable proportion of players act or idle synchronously. Based on a detailed analysis, we find that the design of global events, i.e., random events held by the system, is one possible cause of the phenomenon. A global event can be seen as a special type of "quest" that encourages players to actively join a short-term activity and rewards the participants with experience points. For example, "a horde of monsters appear in the town, and the mayor calls for help from players" would be a typical global event. When such events occur, players near the scene could join forces to eliminate the monsters. Global events are held every 10 or $20 \mathrm{~min}$. An event is finished once the assigned objective has been achieved, which usually takes several minutes. Since a number of players fight in global events and return to their own journeys afterwards, the events can cause flash crowd effects.

To demonstrate the impact of global events, in Fig. 12(b), we depict the average packet rates sampled every $2 \mathrm{~min}$ in a 2 -h interval. We can see that peaks always occur around multiples of $10 \mathrm{~min}$, and therefore confirm the effect of global events which are held every 10 or $20 \mathrm{~min}$. We also observe that a dip occurs following each peak with a distance of 2-3 samples (corresponding to 4-6 min), which may indicate the time that elapses until each global event quietens down. The effects of global events undoubtedly lead to positive temporal dependence in user activities, and therefore locality in client packet arrivals. Though global events do not take place continuously, due to their significance and frequency, they affect the overall temporal dependence of client traffic to some extent. On the other hand, we consider the heavy-tailed ON/ OFF-periods of players activities as another cause of the large-scale correlations in game traffic. We discuss this point in Section 5.3.

\subsection{Periodicity}

Taking a closer look at Fig. 12(b), i.e., focusing on the auto-correlation function over time lags shorter than $1 \mathrm{~s}$, we get the result shown in Fig. 13(a). According to the graph, we find that some form of periodicity exists in client packet arrivals in that higher correlation coefficients occur at lags equal to multiples of $200 \mathrm{~ms}$. However, it is hard to tell whether periodicity actually exists by visually checking the auto-correlation functions, so we employ a frequency domain method to examine the periodicity in client traffic. In contrast to client traffic, server traffic is apparently periodic with a cycle of $200 \mathrm{~ms}$, as shown in Fig. 13(b). This implies the round-based position updates are synchronous for all game clients.

Power spectral density (PSD) is a more direct way to analyze the frequency components in time series. Fig. 14 indicates strong periodicity in traffic in both directions, i.e., multiples of $5 \mathrm{~Hz}$ in server traffic and multiples of $6 \mathrm{~Hz}$ in client traffic. According to the graph, server traffic is more regular than client traffic, as less spikes occur in Fig. 14(b). This is not only because server traffic is more periodic, but also because we obtained the packet trace at the server side; therefore, the client packets were time-stamped after they had travelled over the network, but the server packets were not.

The identified frequency of $5 \mathrm{~Hz}$ of server traffic clearly echoes the large proportion of $200 \mathrm{~ms}$ server packet interarrival times identified in Fig. 6. We found that frequencies with multiples of $5 \mathrm{~Hz}$ also exist, as servers adjust the frequency of position updates based on certain factors, such as the density of neighbors. At the same time, we found that the multiples of $6 \mathrm{~Hz}$ frequency components in client traffic are due to automatically generated commands. Specifically, a player can switch to an "auto-movement" mode by clicking the left mouse button for $2 \mathrm{~s}$, and the character will continuously move toward the mouse cursor until it is switched back to normal mode. Attack actions can also be performed automatically by entering the "auto-attack" mode. In the client's implementation, a timer of corresponding frequency is used to issue movement or attack commands in place of the player, where the frequency is decided by the level and skill of the character, and the weapons it holds. The periodicity should be cancelled out if the clients' timers are not synchronized; however, it still exists. From a discus- 


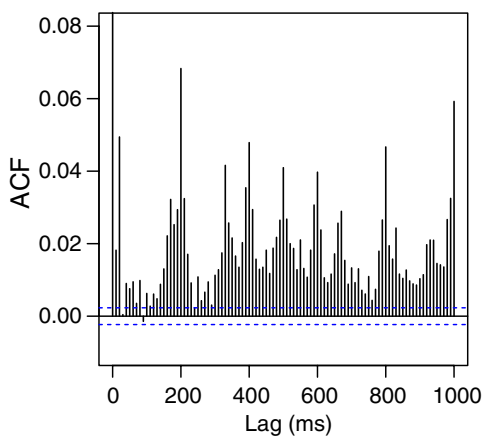

(a) ACF of client packet arrival

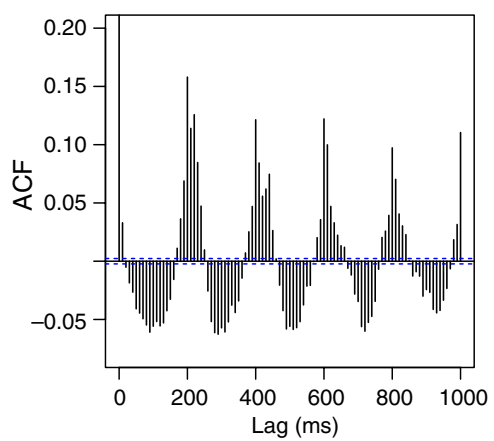

(b) ACF of server packet arrival

Fig. 13. Correlograms of aggregate packet arrivals.

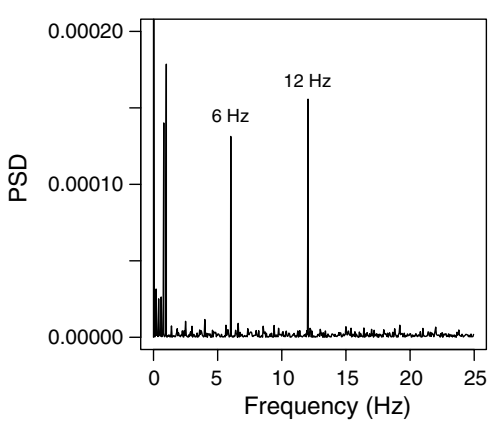

(a) PSD of client packet arrival

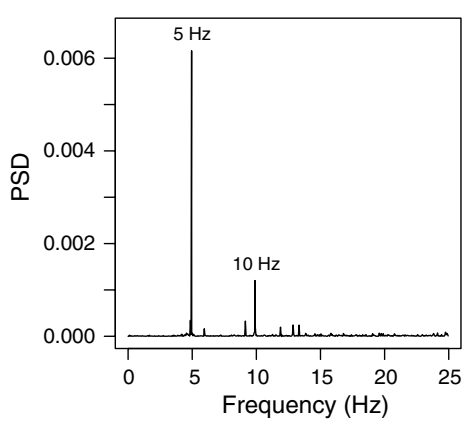

(b) PSD of server packet arrival

Fig. 14. Power spectral density of aggregate packet arrivals.

sion with ShenZhou Online developers, we found that the synchronization of clients' timers is because their initialization is based on the arrival of server packets, which are sent in a burst to all clients. Although this was not the developers' original intention, the design choice ensures that game clients act in a synchronized manner.

The strong frequency components in server traffic imply that a large proportion of server packets are sent in bursts periodically. We believe that bursts of server packets (primarily position updates) are unnecessary and can lead to performance problems. Our conjecture is that server packets sent periodically have a higher probability of being dropped. To verify this hypothesis, we estimate the round-trip times (RTT) and packet loss events in the traces. As the trace was taken at the server side, a roundtrip time is defined as "the time that elapses before a server data packet is acknowledged by an acknowledgement packet sent back from the client." Packet loss events are inferred by packet retransmissions, where a server data packet is considered lost if an identical packet (at the TCP level) is re-transmitted. We first examine whether any pattern exists in the round-trip times by the correlogram and power spectrum. As shown in Fig. 15(a) and (b), the autocorrelation function estimated from the RTT process shows that high correlation coefficients exist at multiples of $200 \mathrm{~ms}$ lags. The power spectrum clearly shows that RTTs are periodic at $5 \mathrm{~Hz}$ and $10 \mathrm{~Hz}$, which is an identical pattern to the frequencies of the position updates. Furthermore, the counting process of packet loss events within each $100 \mathrm{~ms}$ exhibits strong periodicity in the correlogram and power spectrum, as shown in Fig. 15(c) and (d) respectively. Again, the frequency of packet loss echoes that of the server position updates. The phenomenon whereby both RTT and packet loss are correlated with the position updates sent in bursts evidences our conjecture that periodicity rooted in the system degrades network performance.

The common design pattern of network games tends to send out periodic and synchronized state updates, where the periodicity comes from a predefined update frequency, and the synchronization comes from batch message dispatches with a loop for all clients. Furthermore, the synchronization can be propagated into other parts of the system, 


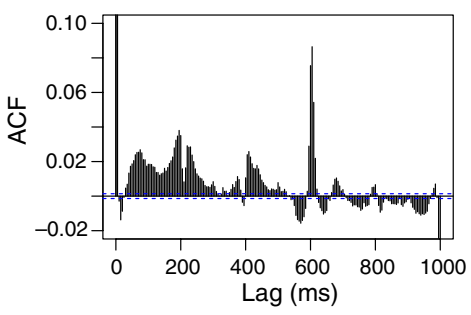

(a) ACF of round trip times

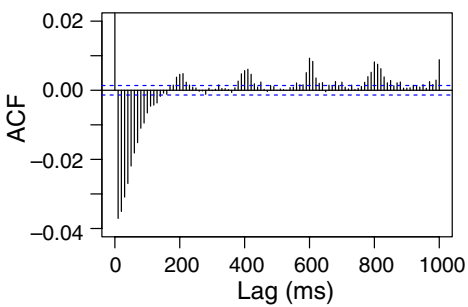

(c) ACF of number of packet loss events

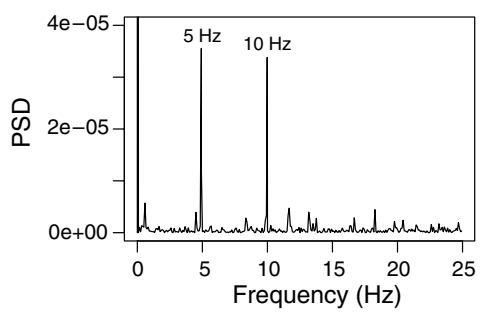

(b) PSD of round trip times

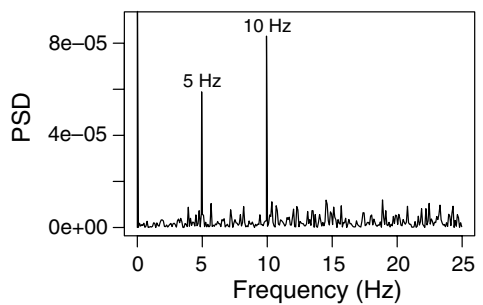

(d) PSD of number of packet loss events

Fig. 15. (a,b) Correlogram and power spectrum of time series formed by the average RTT every 5 ms; (c,d) Correlogram and power spectrum of time series formed by the number of dropped packets every $100 \mathrm{~ms}$.

since certain actions that act as response to commands from the opposite party may release more messages accordingly. We remark that, though this design is relatively simple and intuitive, it can have an adverse impact on network performance.

\subsection{Burstiness and self-similarity}

Earlier, we noted the large-scale correlations and periodicity in aggregate traffic. We now further investigate the variability of aggregate traffic in coarser time scales. As the traffic burstiness of individual game connections has been shown to be inbetween that of Poisson arrivals and TELNET connections (Section 4.5), we first check how their traffic variability varies from flow multiplexing.

To this end, in Fig. 16, we draw variance-time plots for the aggregate game traffic, and the $L B L$ TCP3 TELNET traffic, as well as a simulated trace of the aggregate Poisson arrivals. In this method, the counting process is divided into multiple, consecutive, equal sized, blocks. For each block size, which is 2 through $10^{4}$ in our case, an aggregate process is composed by averaging the values within each block. The variance-time plot is formed by plotting the variances of the aggregated processes versus the aggregation level (block size), on a log $\log$ scale. For comparison between processes with different packet numbers, all processes are normalized to an average rate of $1 \mathrm{pkt} / \mathrm{s}$ before computing their variances over different aggregation levels. We

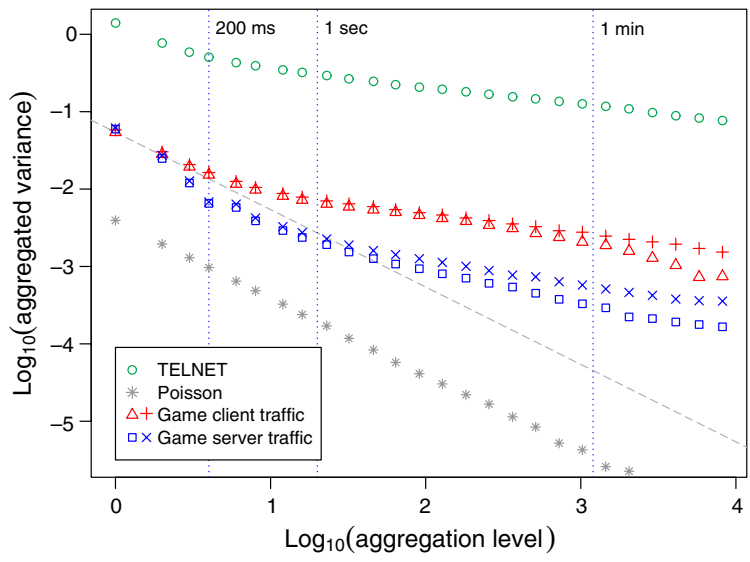

Fig. 16. Variance-time plot for the packet arrival processes. The line from the upper left corner has a slope of -1 .

also ensure the same degree of multiplexing by counting only the longest 250 TELNET connections in the $L B L-T C P 3$ trace, as 250 connections are multiplexed in the sampled game traffic.

Fig. 16 indicates that, on aggregate, game traffic in both directions is much less bursty than TELNET traffic; however, it is more bursty than the aggregate Poisson arrival process, which is multiplexed by 250 i.i.d. exponential inter-arrival processes. The relative burstiness of game traffic compared to the TELNET and Poisson processes is approximately the same with and without flow multiplexing, except that the server traffic of individual connections can 
be smoother than Poisson arrivals in small time scales (see Fig. 11). At aggregation levels $M$ around 4 (equivalent to $200 \mathrm{~ms}$ ), the variances of game server traffic decay more quickly than $1 / M$, indicating that the process has less persistent auto-correlations than Poisson processes, or periodicity exists. We believe this is caused by the periodicity and synchronization between connections rooted in the game's design, which we identified in Section 5.2.

To visually grasp the discrepancy between traffic of different applications, in Fig. 17, we plot the arrival processes corresponding to 1 -s intervals $(M=20)$ for TELNET traffic, game client traffic, and Poisson processes, each with a multiplexing level of 250. As can be seen from the figure, TELNET traffic is much more bursty than the other traffic types. While the Poisson process has a variance of 0.07 and game client traffic has a variance of 3.24, TELNET traffic has a variance of 79.35. This indicates that game traffic is much less bursty than TELNET traffic, and relatively close to Poisson processes in small time scales. The large discrepancy between TELNET traffic and game traffic originates at the connection-level packet interarrivals, because TELNET packet interarrival times are heavy-tailed, whereas game client packet interarrival times are close to exponential. In addition, the periodic mechanisms and synchronization between game clients further reduce the burstiness of game traffic.
The variance-time plot is also a means of testing the scaling property. Except for an initial dip, the aggregated variances of game traffic are approximately linear and the slope of the downtrend is flatter than -1 , which is an indicator of (asymptotic) second-order self-similarity [17]. The Hurst parameter $H$ can be estimated by taking the magnitude of the slope $\beta$ of the best-fit line through the data points with the relation $H=1-\frac{\beta}{2}$. In our case, the Hurst parameters are estimated as about 0.85 for client traffic and about 0.8 for server traffic, both of which suggest long-range dependence and possibly second-order self-similarity.

However, the estimation of Hurst parameters has been shown to be unreliable for certain effects in processes, such as trends, periodicity, and non-stationarity. We can remove trends and non-stationarity by carefully designed sub-sampling and a short sampling interval. Periodicity, however, cannot be easily removed without loss of sampling accuracy, since we do not know whether a packet was sent due to periodic mechanisms or not. As we were aware of the frequency components in the game traffic, mainly $5 \mathrm{~Hz}$ and $6 \mathrm{~Hz}$, we ran a simulation study to investigate the influence of such frequency components on the estimation of the Hurst parameter. In the simulation, a fractional Gaussian noise (FGN) process of the same length and the same mean $(1 \mathrm{pkt} / \mathrm{s})$ as game traffic was generated;
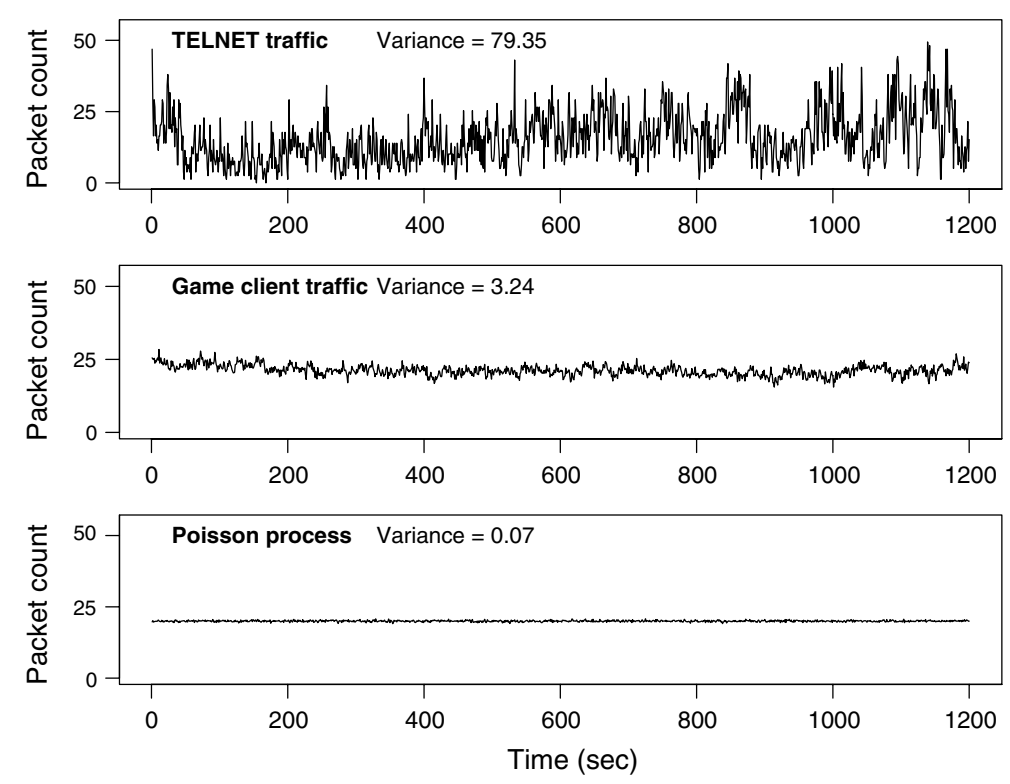

Fig. 17. Comparison of aggregate TELNET traffic, game client traffic, and Poisson processes. 
after that, a $5 \mathrm{~Hz}$ noise, which is a uniform random variable with a range of $(0.5,1)$ packet, was added. To mimic the real-life randomness in the periodic release of packet bursts, i.e., the intervals between periodic bursts are not exactly the same, the time slots where noise was added were disturbed by a zero-mean normal random variable with a standard deviation of $40 \mathrm{~ms}$. To determine the effect of periodic noise, we adopted wavelet multiresolution anal$y$ sis [18] to examine and compare the scaling property of the perturbed FGN processes and game traffic. As shown in Fig. 18(a), the energy of the perturbed FGN process deviates substantially from that of the original FGN process over time scales smaller than $200 \mathrm{~ms}$, where the energy increases significantly due to $5 \mathrm{~Hz}$ noise. Turning to Fig. 18(b), we can see that the trend of wavelet energy of game traffic shows the same effect observed in the FGN simulation, where the non-linear part at small time scales is attributable to periodicity. Thus, we can safely disregard time scales where the energy is disturbed by the periodicity, and focus only on time scales coarser than $200 \mathrm{~ms}$, i.e., the fifth octave and higher. As a result, we observe that energy at those larger scales $(>200 \mathrm{~ms})$ is approximately linear, which also suggests self-similarity.

Periodicity affects other estimation methodologies in a similar manner. In Fig. 19, we provide the Hurst estimates for game traffic in both directions and simulated FGN processes over different aggregation levels. The Hurst estimators we use include the Whittle estimator, aggregated variance method, R/S (rescaled-adjusted range plot) method, periodogram method, and wavelet method. In the upper plot, we show that the Hurst estimate is not stable until the aggregation level is greater than 50 $(\approx 2.5 \mathrm{~s})$ for the Whittle estimator, which is usually considered robust. In the middle and lower plots, the Whittle estimate, as well as its $95 \%$ confidence intervals, for game traffic in both directions over aggregation levels up to $1000(\approx 50 \mathrm{~s})$, are provided. Here, the Hurst estimates, except that of the Whittle estimator, are fixed at aggregation level 50. As can be seen, the Whittle's Hurst estimate remains constant over a wide range of time scales. The Hurst estimates by other methods generally agree with each other, and are roughly within the confidence intervals of the Whittle estimates, at least for large aggregation levels. As a result, the Hurst parameter we estimated is within $(0.85,0.9)$ for game client traffic, and within $(0.8,0.85)$ for server traffic. Though not listed here, the results drawn from all 2-h intervals of our game traces are consistent with the above findings.

With regard to the question of why self-similarity exists in game traffic, we find that the heavy-tailed ON/OFF source-model provides a reasonable explanation [17]. We define ON- and OFF-periods as corresponding to a player's activity and idle periods respectively; consequently, the game client for each connection can be modeled as a strictly alternating ON/OFF source. Since game clients send certain packets, such as keep-alive messages or information queries about the environment even when a character is completely idle, we cannot simply treat a client data packet as an action issued by the player. To examine the distribution of ON/OFF periods for individual connections, we compute the average client data packet rates in 4-s intervals. Based on the histograms of the packet rates sampled, we choose a threshold of $1.5 \mathrm{pkt} / \mathrm{s}$ to determine whether a player is "active" or "idle" in a certain interval. The sampling interval is chosen arbitrarily so that it can capture the active and idle behavior of players' activity; however, we find that different intervals, e.g., 1-s through 10-s intervals, lead to similar results.

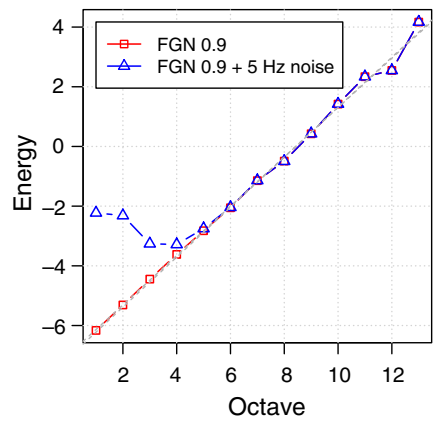

(a) Simulated FGN processes

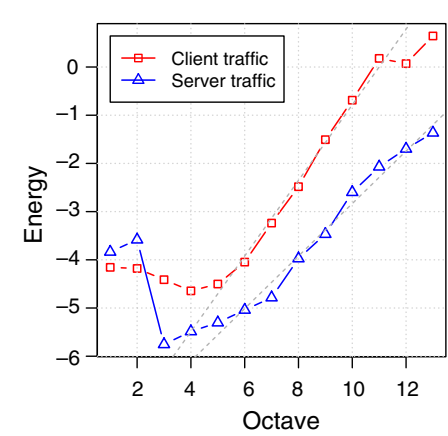

(b) Game packet arrival processes

Fig. 18. The scaling property using wavelet multiresolution analysis. 


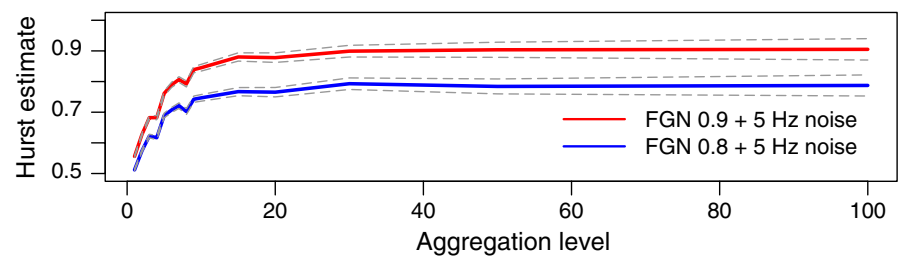

(a) FGN processes with noise

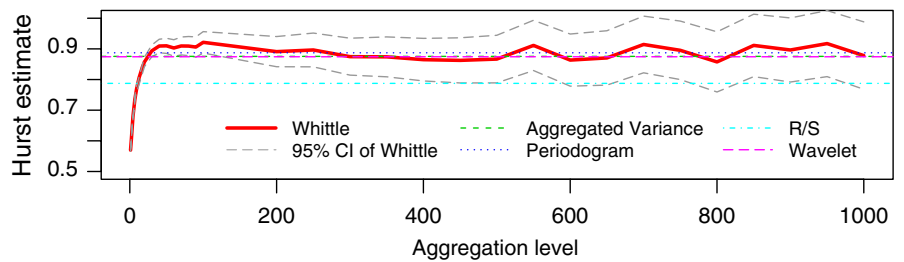

(b) Game client packet arrivals

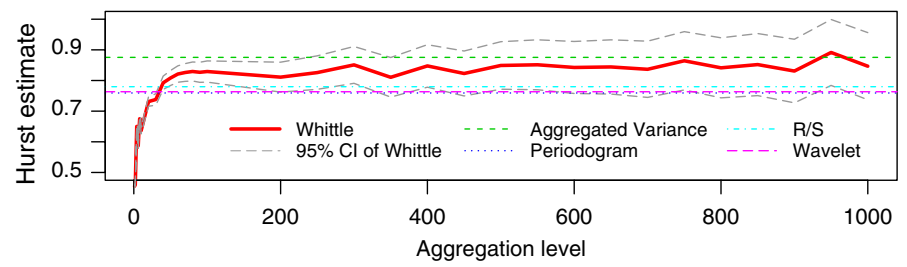

(c) Game server packet arrivals

Fig. 19. Hurst estimates for simulated FGN processes and game traffic in both directions.

A distribution of a random variable $X$ is heavytailed if

$P[X>x] \sim x^{-\alpha} \quad$ as $x \rightarrow \infty$,

where the power-law exponent, $\alpha$, is within the range $0<\alpha<2$. According to [17], the aggregate traffic of multiplexed connections is self-similar if the traffic within individual connections can be modelled as ON/OFF periods, and either ON- or OFF-periods are heavy-tailed distributions with $1<\alpha<2$. After the ON- and OFF-periods for each connection have been computed, we can then estimate the heaviness of their tail distributions, i.e., whether the tail distributions of ON/OFF-periods conform to $1<\alpha<2$.

However, in our traces, ON/OFF periods were censored due to the beginning and termination of the measurement, i.e., we did not observe the full span of some periods, so the exact length of each of those periods is unknown. Furthermore, since long periods are more likely to be censored, we cannot simply estimate the tail heaviness from observed times or only uncensored periods, as this will lead to underestimating tail heaviness. While the Hill estimator for right-censored data is available [19], for randomly-censored data (which is the case with our ON/OFF-periods), it remains to be extended. Therefore, we adopt a simple heuristic method to estimate $\alpha$ based on the Kaplan-Meier survival curves [20] (a brief introduction is provided in Section 6.2), i.e., we estimate the complementary cumulative distribution function (CCDF) for both censored and uncensored observations. Taking the logarithm on both sides of Eq. (1), we obtain that the logarithm of a survival curve of $X$ would be linear with a slope of $-\alpha$ if $X$ follows the power law with exponent $\alpha$. By this method, we can obtain an $\alpha$-estimate based on the slope of the line fitted to the $\log -\log$ survival curve. In Fig. 20, we draw log-log survival curves for the $\mathrm{ON}$ - and OFF-periods of some connections, where the survival function of an exponential random variable is also provided for reference. In the plots, we observe that both $\mathrm{ON}$ - and OFF-periods, especially OFF-periods, tend to be heavy-tailed in that their tail distribution is approximately linear. We can see that the distribution of game session times declines more slowly than the exponential distribution, especially in the tail portion.

In order to check the heavy-tailedness of $\mathrm{ON} /$ OFF-periods, we extracted 223 connections that 


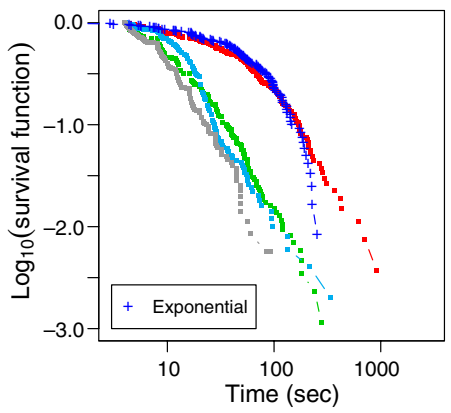

(a) ON-periods

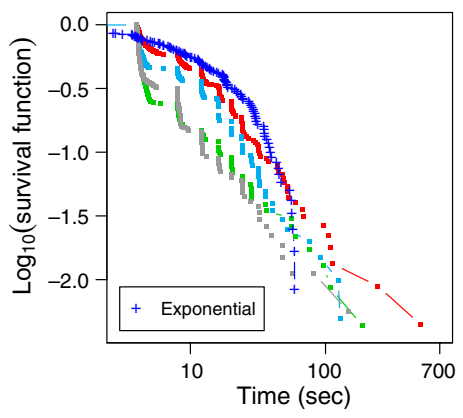

(b) OFF-periods

Fig. 20. Log-log survival curves of ON/OFF-periods within connections.

spanned more than $2 \mathrm{~h}$ and contained more than 150 ON/OFF-periods. To estimate $\alpha$, we used a linear regression on the tail $20 \%$ of the observations. To visually grasp the $\alpha$ estimates for different connections, we categorized the $\alpha$-estimates in intervals of $(0, .75),(.75,1.25),(1.25,1.75), \quad(1.75,2.25)$, $(2.25,2.75),(2.75, \infty)$ and plotted the corresponding ranges for $\mathrm{ON}$ - and $\mathrm{OFF}$-periods respectively, as shown in Fig. 21. We can see from the figure that the $\alpha$-estimates for OFF-periods cover the whole interval $(1,2)$, which is evidence of heavy-tailedness. About $35 \%$ of sessions have $\alpha$-estimates outside the range. This is reasonable, because some players never take a long break during their online time, e.g., users at an Internet café. Though the number of sessions without heavy-tailed OFF periods is not insignificant, the majority of sessions do have heavy-tailed OFF periods and can have a substantial impact on the overall traffic characteristics. On the other hand, the $\alpha$-estimates for ON-periods primarily cover the range $(1.5,2.5)$, which has generally lighter tails than OFF-periods and may not be heavy-tailed for a number of connections. This behavior is plausible, since OFF-periods (idle periods) can be arbitrarily long, as players can just leave their computers. ON-periods, however,

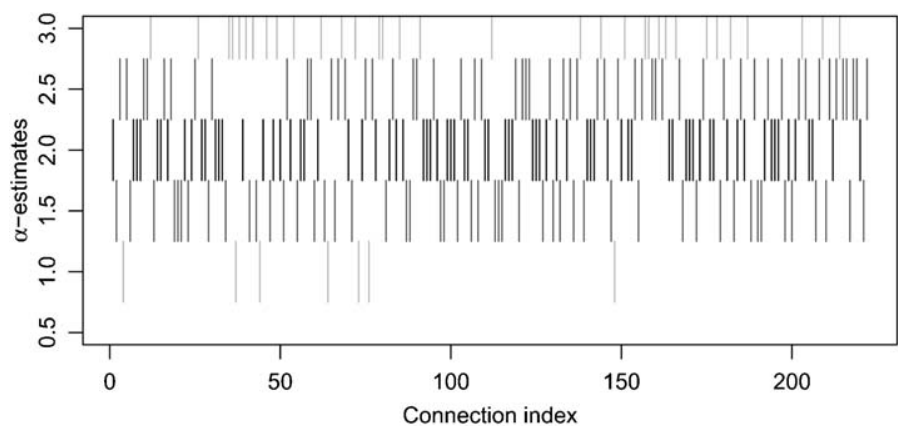

(a) ON-periods

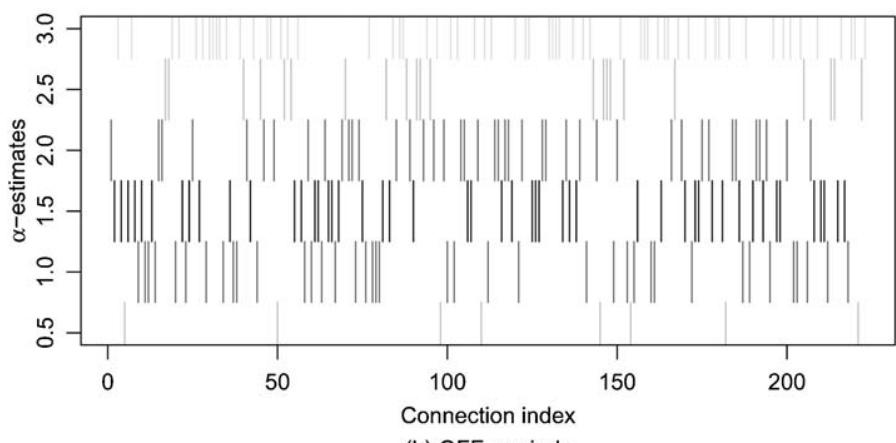

(b) OFF-periods

Fig. 21. Summary plots of the ranges for the $\alpha$-estimates for ON-periods and OFF-periods of 223 connections. 
require players to be active for a long time without a break, which of course is unlikely to be too long given the physical limits of human beings. In the ON/OFF source models, the Hurst parameter is decided by the minimum of $\alpha$ of ON- and OFF-periods; thus, the OFF-periods with $1<\alpha<2$ can be seen as the primary cause of the self-similarity observed in game traffic.

\section{Session characteristics}

In ShenZhou Online, the game world is partitioned into a number of maps, provided by one or more map servers. When a character moves across map boundaries, if the new map is provided by a different map server, the game client will disconnect from the original map server and establish a connection with the new map server. The design naturally leads to two types of session: (1) map session: the period a character remains on a map; and (2) game session: the period a player remains in a game. The map session is of interest to us, since it is semantically equivalent to a TCP connection, and the modeling of TCP connections is relevant in traffic modeling. On the other hand, the game session describes how long a player stays in a game, which is important in user behavior modeling. For the sake of brevity, we use "sessions" to denote both map sessions and game sessions.

In the following, we explore how often players switch maps and join a game, i.e., the interarrival times of map sessions and game sessions, respectively. We also investigate the periods that players remain on a map and in a game, i.e., the duration of map sessions and game sessions, respectively.

\subsection{Interarrivals}

Fig. 22 plots the CCDF of the interarrival times of map sessions and game sessions, respectively, with a log-linear scale. We can see that both map sessions and game sessions are consistent with the exponential distribution, as their CCDFs are nearly straight on the graph.

In order to evaluate whether the session interarrivals follow a Poisson arrival process, we use the statistical tests proposed in [16]. The method tests two aspects of session interarrivals: whether they are exponentially distributed, and whether they are independent. We first test the interarrivals for an exponential distribution using the Anderson-Darling test recommended by Stephens in [21]. Then,

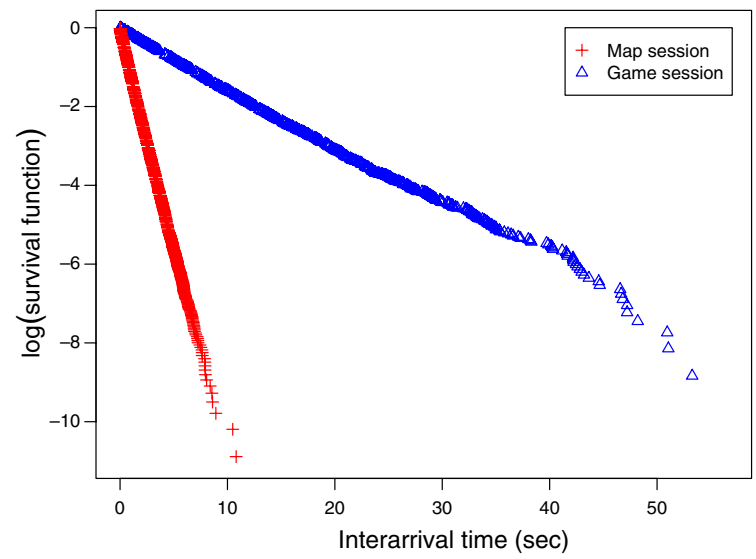

Fig. 22. Session interarrival times in a log-linear scale.

we test the independence of interarrivals by the auto-correlation functions. For independent interarrivals, the magnitude of the corresponding auto-correlation function should not be too large, and there should be approximately equal numbers of positive and negative auto-correlations (refer to [16] for details of the tests).

Because of the significant time-of-the-day effect, we cannot expect session interarrivals from the entire trace to fit a single homogeneous Poisson model. Instead, we apply the Poisson conformance test to packet interarrivals in each 1-h interval. With a significance level of 0.05 for all tests, we find that game sessions within 1 -h intervals conform to homogeneous Poisson processes. The result is reasonable, since the game sessions were manually initiated by players. On the other hand, map sessions do not pass the independence test, even with an interval of $10 \mathrm{~min}$. We consider that the temporal dependence of map session arrivals arises from the common behavior of team play, i.e., players usually team up in support of each other. Because team players move on a map together, a number of map sessions will be initiated at approximately the same time when a group of players moves across a map boundary. In recent years, the design of MMORPGs has tended to encourage even forced players to team up to solve quests in a game. Therefore, we believe the dependent arrivals of map sessions is not a unique feature of ShenZhou Online, i.e., it is shared by many MMORPGs.

\subsection{Duration}

Because of the setup of our traffic measurement, some players had been in the game before the trace 
Table 2

Summary of map/game sessions

\begin{tabular}{|c|c|c|c|c|c|}
\hline & \# Sessions & \# Cens. & Minimum (s) & $\operatorname{Median}^{\mathrm{a}}(\min )$ & Maximum (min) \\
\hline \multicolumn{6}{|l|}{ Map } \\
\hline $\mathscr{N}_{1}$ & 57,689 & $3609(6 \%)$ & 16 & 4.4 & 475 \\
\hline $\mathscr{N}_{2}$ & 54,299 & $1839(3 \%)$ & 12 & 4.2 & 716 \\
\hline Total & 111,988 & $5448(5 \%)$ & 12 & 4.3 & 716 \\
\hline \multicolumn{6}{|l|}{ Game } \\
\hline $\mathscr{N}_{1}$ & 7597 & $3331(44 \%)$ & 27 & 122 & 487 \\
\hline $\mathscr{N}_{2}$ & 7543 & $1774(24 \%)$ & 22 & 86 & 729 \\
\hline Total & 15,140 & $5105(34 \%)$ & 22 & 100 & 729 \\
\hline
\end{tabular}

${ }^{\text {a }}$ Estimated with the Kaplan-Meier curve (Eq. (2)).

started, and some left after the trace terminated; therefore, we do not know exactly how long those players stayed in game. We define a session as censored if only part of it was observed by our network monitor. As a significant proportion of observed sessions were censored, statistics only computed from observed time will lead to underestimation. Therefore, we use statistical methods in survival analysis, which are capable of handling censored observations, to compute statistics related to session duration.

By the conventions of survival analysis, we denote a player's departure as an event or a failure. A survival function is commonly used to describe the lifetime pattern of an object or a set of observations. In our context, the survival function is defined as:

$$
\begin{aligned}
S(t) & =\operatorname{Pr}(\text { a session that survives longer than time } t) \\
& =1-\operatorname{Pr}(\text { a session that fails before, or at time } t) \\
& =1-F(t),
\end{aligned}
$$

where $F(t)$ is the cumulative distribution function (CDF) of session times. A standard estimator of the survival function, proposed by Kaplan and Meier [20], is called the Product-Limit estimator or the Kaplan-Meier estimator. Suppose there are $n$ distinct session times $t_{1}, t_{2}, \ldots, t_{n}$ in ascending order such that $t_{1}<t_{2}<\cdots<t_{n}$; and that at time $t_{i}$, there are $d_{i}$ events and $Y_{i}$ active sessions. The survival function of the random variable $T$ for all values of $t \leqslant t_{n}$ is then defined as follows:

$$
\hat{S}(t)=\prod_{t_{i} \leqslant t} \operatorname{Pr}\left[T>t_{i} \mid T \geqslant t_{i}\right]= \begin{cases}1 & \text { if } t<t_{1}, \\ \prod_{t_{i} \leqslant t}\left[1-\frac{d_{i}}{Y_{i}}\right] & \text { if } t_{1} \leqslant t .\end{cases}
$$

Accordingly, the $p$ th quantile of the lifetime, $t_{p}$, can be obtained by

$t_{p}=\inf \{t: \hat{S}(t) \leqslant 1-p\}$.
With Eq. (2), we can estimate the median session time for a group of sessions by $t_{0.5}$, as the median times listed in Table 2.

The hazard function gives us a direct way to check the lifetime pattern of sessions. The hazard function, or the hazard rate, also known as the conditional failure rate in reliability engineering, or the intensity function in stochastic processes, is defined by

$h(t)=\lim _{\Delta t \rightarrow 0} \frac{\operatorname{Pr}[t \leqslant T<t+\Delta t \mid T \geqslant t]}{\Delta t}$.

The hazard function gives the instantaneous rate at which failures occur for observations that have survived at time $t$. In the context of game sessions, the quantity $h(t) \Delta t$ may therefore be seen as the approximate probability that a player who has been in a game for time $t$ will leave the game in the next $\Delta t$ period, given that $\Delta t$ is small. We depict the hazard functions estimated for game sessions in Fig. 23. The distinct hazard functions for game sessions on weekdays and weekends clearly show a day-of-theweek effect. Furthermore, both hazard functions

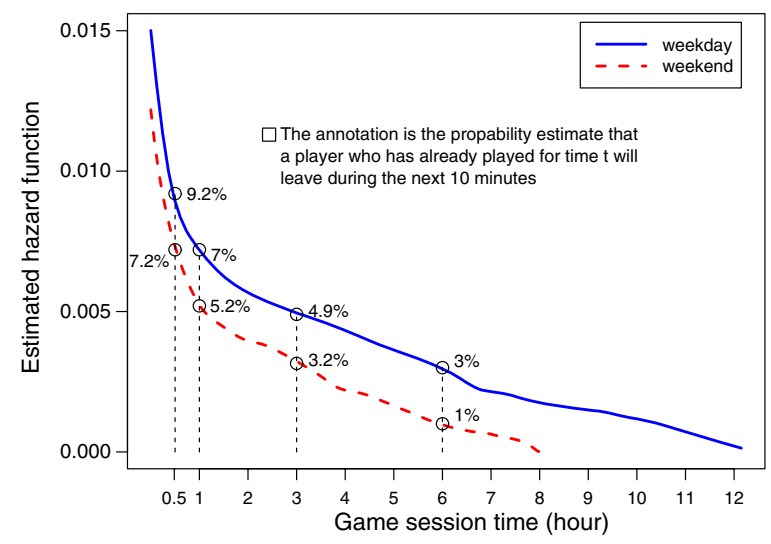

Fig. 23. Estimated hazard function for game sessions. 


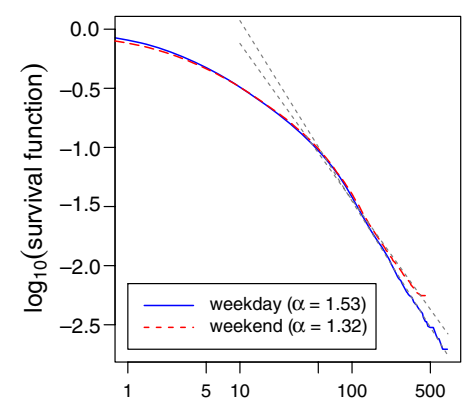

(a) Map session time (min)

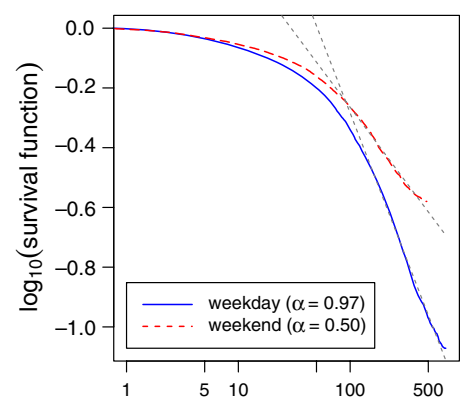

(b) Game session time (min)

Fig. 24. Log-log survival curves for estimating the tail-heaviness of session duration.

present continuously downward trends. To be more specific, based on the computed weekday hazard function, the probability that a player will leave a game within a short time $(10 \mathrm{~min})$ when he has been in the game for $30 \mathrm{~min}, 1 \mathrm{~h}, 3 \mathrm{~h}$, and $6 \mathrm{~h}$ is approximately $9.2 \%, 7 \%, 4.9 \%$, and $3 \%$, respectively. The decreasing failure rate indicates that a player who has played for a long time tends to stay longer, which is a remarkable feature of heavy-tailed distributions. The hazard functions of map sessions (not provided here) exhibit similar behavior of constantly downward trends.

To verify the heavy-tailedness of session times, we plot the survival functions of the session times on a log-log scale, as shown in Fig. 24. A simple linear regression of the tail distributions indicates that $1.3<\alpha<1.6$ for map sessions and $0.5<\alpha<1$ for game sessions: both types of session are heavytailed, where game sessions have heavier tails with a statistically infinite mean, i.e., the expected duration of game sessions is divergent.

The lifetime pattern of the game sessions we observed was very different from earlier studies for FPS (First-Person Shooting) games [22,23], in which the session times were not heavy-tailed. We attribute this discrepancy to the difference between game genres. FPS games, which are basically roundbased, force players to break off following each round and give players a chance to regain consciousness of the real world. On the other hand, the adventures in MMORPGs are continuous and endless, and no explicit mechanism exists to give players a pause. The heavy-tailed game sessions can be seen a consequence of so-called MMORPG addiction. As the Daedalus Project [24] remarks: "about 50\% of MMORPG players would consider themselves addicted to the games." To explain the addiction, Yee [24] proposed three main attraction factors of MMORPGs, namely, the elaborate rewards cycle, a network of relationships, and the immersive nature of virtual environments, which encourage time investment and personal attachment. We believe such factors are the key reasons that MMORPGs are so popular and also explain the addictive lifetime pattern of game playing times.

\section{Conclusion}

In this paper, we have presented an analysis of a packet trace from ShenZhou Online, a mid-sized commercial MMORPG. The trace reveals that MMORPG traffic is very different from the traffic of dominant Internet applications, such as file transfers and web surfing. In summary, we have identified the following important properties of MMORPG traffic: (1) tiny packets, (2) periodicity, (3) temporal dependence of packet arrivals within connections and aggregate traffic, (4) self-similarity, and (5) heavy-tailed session duration. We have also offered physical explanations for the observed traffic characteristics based on the following idiosyncratic user behavior in MMORPGs: (1) the diversity of user behavior, (2) the clustering nature of user inputs, (3) the flash crowd effect, (4) the practice of team play, and (5) heavy-tailed active/idle user activity periods.

With regard to the question of whether or not TCP is suitable for MMORPGs, we observe a significant amount of overhead from the TCP/IP header and acknowledgments. The cost is noteworthy: $\mathrm{TCP} / \mathrm{IP}$ headers comprise $73 \%$ of transmitted bytes, where pure TCP acknowledgement packets are responsible for $30 \%$. Note that the former includes the latter. This suggests that TCP with a sizeable header and positive acknowledgement mechanism is overkill for applications such as MMORPGs. The effect of TCP on the end-to-end delay of MMORPGs will be a good topic for further study. 


\section{Acknowledgements}

This work would not have been possible without the extensive traffic trace of ShenZhou Online. The authors are much indebted to the following people who helped us gather the trace: Tsing-San Cheng, Lawrence Ho, Chen-Hsi Li, and especially to YenShuo $\mathrm{Su}$, who between them made the datasets available. The authors also wish to thank Wu-chang Feng and the anonymous referees for their constructive criticisms.

\section{References}

[1] K.-T. Chen, P. Huang, C.-Y. Huang, C.-L. Lei, Game traffic analysis: an MMORPG perspective, in: NOSSDAV'05: Proceedings of the International Workshop on Network and Operating Systems Support for Digital Audio and Video, ACM Press, 2005, pp. 19-24.

[2] B.S. Woodcock, An analysis of MMOG subscription growth-version 18.0. Available from: <http://www.mmogchart.com/>.

[3] S. McCreary, K. Claffy, Trends in wide area IP traffic patterns: a view from Ames Internet exchange, in: Proceedings of 13th ITC Specialist Seminar on Measurement and Modeling of IP Traffic, 2000, pp. 1-11.

[4] ShenZhou Online, UserJoy Technology Co., Ltd. Available from: $<$ http://www.ewsoft.com.tw/ $>$.

[5] W.C. Feng, F. Chang, W.C. Feng, J. Walpole, A traffic characterization of popular on-line games, IEEE/ACM Trans. Network. 13 (3) (2005) 488-500.

[6] R.A. Bangun, E. Dutkiewicz, G.J. Anido, An analysis of multi-player network games traffic, in: Proceedings of the 1999 International Workshop on Multimedia Signal Processing, Copenhagen, Denmark, 1999, pp. 3-8.

[7] M.S. Borella, Source models of network game traffic, Comput. Commun. 23 (4) (2000) 403-410.

[8] J. Färber, Network game traffic modelling, in: NetGames '02: Proceedings of the 1st Workshop on Network and System Support for Games, ACM Press, 2002, pp. 5357.

[9] V. Jacobson, C. Leres, S. McCanne, tcpdump, 1989. Available from: $<$ ftp://ftp.ee.lbl.gov $>$.

[10] R. Braden, Requirements for Internet Hosts - Communication Layers, RFC 1122 (Standard), October 1989.

[11] C. Partridge, P.P. Carvey, E. Burgess, I. Castineyra, T. Clarke, L. Graham, M. Hathaway, P. Herman, A. King, S. Kohalmi, T. Ma, J. Mcallen, T. Mendez, W.C. Milliken, R. Pettyjohn, J. Rokosz, J. Seeger, M. Sollins, S. Storch, B. Tober, G.D. Troxel, A 50-Gb/s IP router, IEEE/ACM Trans. Network. 6 (3) (1998) 237-248.

[12] N. Sheldon, E. Girard, S. Borg, M. Claypool, E. Agu, The effect of latency on user performance in Warcraft III, in: NetGames '03: Proceedings of the 2nd Workshop on Network and System Support for Games, ACM Press, 2003, pp. 3-14.

[13] R. Gusella, Characterizing the variability of arrival processes with indexes of dispersion, IEEE J. Select. Areas Commun. 9 (2) (1991).
[14] P. Danzig, J. Mogul, V. Paxson, M. Schwartz, The Internet Traffic Archive. Available from: $<$ http://ita.ee.lbl.gov/ $>$.

[15] R. Jain, The Art of Computer Systems Performance Analysis, John Wiley \& Son, Inc., New York, 1991.

[16] V. Paxson, S. Floyd, Wide area traffic: the failure of Poisson modeling, IEEE/ACM Trans. Network. 3 (3) (1995) 226244.

[17] W. Willinger, M.S. Taqqu, R. Sherman, D.V. Wilson, Selfsimilarity through high-variability: statistical analysis of Ethernet LAN traffic at the source level, IEEE/ACM Trans. Network. 5 (1) (1997) 71-86.

[18] P. Abry, D. Veitch, Wavelet analysis of long-range-dependent traffic, IEEE Trans. Inform. Theory 44 (1) (1998) 2-15.

[19] J. Beirlant, A. Guillou, Pareto index estimation under moderate right censoring, Scand. Actuarial J. 2 (2001) 111125.

[20] E.L. Kaplan, P. Meier, Nonparametric estimation from incomplete observations, J. Amer. Statist. Assoc. 53 (1958) 437-481.

[21] R.B. D'agostino, M.S. Stephen (Eds.), Goodness-of-Fit Techniques, Marcel Dekker, New York and Basel, 1986.

[22] T. Henderson, S. Bhatti, Modelling user behaviour in networked games, in: MULTIMEDIA'01: Proceedings of the Ninth ACM International Conference on Multimedia, ACM Press, 2001, pp. 212-220.

[23] F. Chang, W. chang Feng, Modeling player session times of on-line games, in: NetGames '03: Proceedings of the 2nd Workshop on Network and System Support for Games, ACM Press, 2003, pp. 23-26.

[24] N. Yee, The Daedalus project. Available from: <http:// www.nickyee.com/daedalus $>$.

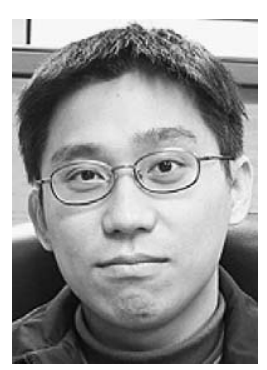

Kuan-Ta Chen received his B.S. and M.S. in Computer Science from National Tsing Hua University, Hsinchu, Taiwan, in 1998 and 2000, respectively. $\mathrm{He}$ is currently pursuing the Ph.D. degree in the Department of Electrical Engineering, National Taiwan University, Taipei, Taiwan. His research interests focus on various aspects of network games, including traffic characterization, communication protocols, quality of service, human factors analysis, and cheating.

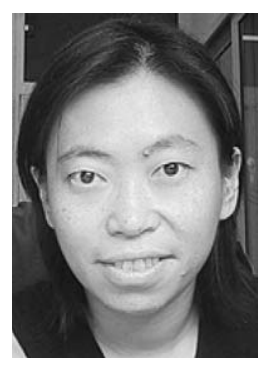

Polly Huang received her B.S. in Mathematics from the National Taiwan University, Taipei Taiwan, in 1993 and her Ph.D. in Computer Science from University of Southern California, Los Angeles USA, in 1999. She is currently an assistant professor at the Department of Electrical Engineering and the Institute of Networking and Multimedia of National Taiwan University. Prior to joining NTU, she worked in the Computer Engineering and Networks Laboratory (TIK) of Swiss Federal Institute of Technology (ETH Zurich) as a post-doctoral research scientist. She is interested in the design, analysis, and application of communication systems in general. 


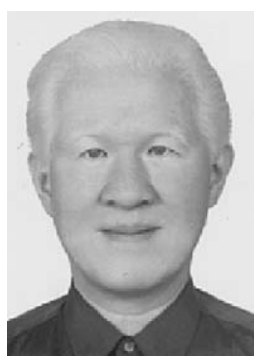

Chin-Laung Lei received his B.S. degree in Electrical Engineering from National Taiwan University in 1980, and his Ph.D. degree in Computer Science from the University of Texas at Austin in 1986. From 1986 to 1988 , he was an assistant professor in the Computer and Information Science Department at the Ohio State University, Columbus, Ohio, USA. In 1988 he joined the faculty of the Department of Electrical Engineering, National Taiwan University, where he is now a professor. His current research interests include computer and network security, cryptography, parallel and distributed processing, design and analysis of algorithms, and operating system design.

$\mathrm{He}$ is a member of the Institute of Electrical and Electronics Engineers and the Association for Computing Machinery. 